\title{
Using aircraft as wind sensors for estimating accurate wind fields for air traffic management applications
}

\author{
Laura Hernando Guadaño, Rosa M Arnaldo Valdés and \\ Francisco J Sáez Nieto
}

\begin{abstract}
A study that examines the use of aircraft as wind sensors in a terminal area for real-time wind estimation in order to improve aircraft trajectory prediction is presented in this paper. We describe not only different sources in the aircraft systems that provide the variables needed to derivate the wind velocity but the capabilities which allow us to present this information for air traffic management applications. Based on wind speed samples from aircraft landing at Madrid-Barajas airport, a real-time wind field will be estimated using a data processing approach through a minimum variance method. Finally, the accuracy of this procedure will be evaluated for this information to be useful to air traffic control.
\end{abstract}

\section{Keywords}

Wind speed, spatial interpolation, atmospheric sensor, flight data

\section{Background and motivation}

The study of the atmosphere behavior has been of particular importance both in SESAR ${ }^{1}$ and NextGen ${ }^{2}$ programs, where the current air traffic management (ATM) system is undergoing a profound transformation to the new paradigms both in Europe and the USA, respectively, to guide and track aircraft more precisely on more efficient routes.

The key element of the future ATM system is based on a fundamental shift in paradigm to four-dimensional (4-D) trajectory management'. ${ }^{3}$ Negotiated trajectories expressed in four dimensions (three spatial dimensions plus time) will satisfy many of the airline preferences (particularly regarding fuel and time consumptions reduction) but will include additional constraints due to limited airspace and airport capacity, in order to achieve an accurate trajectory prediction to produce flyable, efficient and de-conflicted trajectories for all involved aircraft.

Using computational tools to simplify the tasks of human operators involved in the scheme will help to reduce temporal target windows to be met during the flight execution. One example of such tools that could be to make available a real-time wind field estimation for the airport surrounded area not only to both the Air Traffic Control (ATC) and but also to the aircraft.

Uncertainty is a fundamental characteristic of weather phenomena which is transferred to separation assurance, flight path de-confliction and flight planning applications. In this respect, the wind is a key factor regarding the prediction of the future position of the aircraft, so that having a deeper and accurate knowledge of wind field will reduce ATC uncertainties.

The wind information currently is not available for air traffic control, except for aerodrome ATC where it is supplied by local sensors owned by the corresponding national meteorological agency. Furthermore, for different purposes, including flight planning, this agency monitors the Earth's environment and forecasts atmosphere behavior providing reliable and timely information. Its forecasts are based on data from atmospheric observations (balloon, satellite, and aircraft data) taken at various locations worldwide, and numerical weather prediction (NWP) models based on the solution of the "equations of motion" for a fluid: conservation of mass (both of air and moisture), conservation of momentum (Newton's laws), and conservation of thermal energy (thermodynamics). Inaccuracies during spin-up period, spurious behavior of the model during the

School of Aeronautical Engineering, Technical University of Madrid, Madrid, Spain

\section{Corresponding author:}

Laura Hernando Guadaño, Department Aerotecnia, School of Aeronautical Engineering, Technical University of Madrid, Plaza del Cardenal Cisneros, 3, Madrid 28040, Spain.

Email: laura.hernando@upm.es 
first time-steps of the integration, decrease with time, so the minimum-to-maximum spin-up time varies depending on the model from $4-6 \mathrm{~h}^{4}$ to $12 \mathrm{~h} .{ }^{5}$ Therefore, forecasted wind data suffer from small measurement rate with respect to location and time.

The purpose of this article is to describe a new and operationally useful technique intended to provide adequate and direct real-time atmospheric winds fields based on on-board aircraft data. Computationally efficient methods have been developed to estimate horizontal wind components from aircraft ( $V_{\mathrm{GS}}, V_{\mathrm{CAS}} / V_{\mathrm{TAS}}$ ), pressure, and temperature data. These wind data are utilized to estimate a realtime wind field.

This method could be fed by commercial aircraft utilizing their currently available data sources and computational capabilities, and providing them to ATM system where the proposed method could be run. Computed wind velocities, or ground and true airspeeds, would then be broadcasted, for example, via the Aircraft Communication Addressing and Reporting System (ACARS), ADS B out messages, or Mode $\mathrm{S}$. This new source will help updating the wind information furnished in PAMs (meteorological automatic vertical forecast), Meteorological Aerodrome Report (AIRMETs), and Significant Meteorological Information (SIGMETs) reports.

In this paper, we focus on the assessment for required conditions for estimating horizontal wind velocity, the extension to $3-\mathrm{D}$ will be taken up at a later stage. The available data, wind derivation procedure, filtering, and limitations are described in next section. Then, the atmospheric behavior and the equations of fluid mechanics used for atmosphere modeling are described. An interpolation method based on a minimum variance estimator, using two distinct procedures, real and complex 2-D variables is presented in the following section. Below it is shown that the results obtained from flight data recorder (FDR) data available and this lead us to use three wind scenarios that have been considered to test the proposed interpolation methods in next section. Subsequently, wind scenarios results obtained through the two above mentioned interpolation methods are described, and finally, the discussion is presented along with the concluding remarks made in last section.

\section{Methodology}

\section{Data available from the aircraft}

Different on-board systems can provide data needed to derivate the wind velocity vector such as GPS plus air data computer (ADC) ${ }^{6}$ this information is usually stored in the $\mathrm{FDR}^{7,8}$ quick access recorder (QAR), 9,10 and broadcasted by mode $S$ radar system, ${ }^{11,12}$ ACARS, ${ }^{13} 15$ or ADS-B messages, ${ }^{16}$ which receive their inputs from the flight data acquisition unit (FDAU).
The FDAU collects sensor signals (approximately 2500 parameters) and sends parallel data signals to both the FDR and the QAR. The OQAR equipped with an optical disk can store up to $300 \mathrm{~h}$ of flight data, whereas the FDR uses a $25-\mathrm{h}$ looptape. The QAR tapes or disks are replaced at the end of each day or sometimes after a period of several days depending on the media capacity, and they are sent to the carrier offices for analysis.

As different electronic equipment and systems need to be interconnected, the digital information transfer system (DITS) provides the electrical and data characteristics and protocols, as well as the units, ranges, resolutions, refresh rates, number of significant bits, etc. for the data to be transferred to other systems. Depending on the type of data, they are transmitted at intervals ranging from 10 to $20 \mathrm{~ms}$ as the track angle rate, or from 125 to $250 \mathrm{~ms}$ or 250 to $500 \mathrm{~ms}$ depending for the ground speed, depending on the source. ${ }^{17}$

In this work, the FDR data have been supplied by airlines in the frame of a more complex study through a FDR sample for some aircraft. The aircraft types considered include A340, A340-642, A319-111, and A321-211. The parameters measured on board and stored vary from type to type. Basically, the FDR data can be grouped into several categories; viz., inertial data (e.g. position, groundspeed, and accelerations), attitude data (e.g. pitch and roll) and attitude rates (if available), and aerodynamic data (e.g. calibrated airspeed, true air speed (TAS), Mach number, etc.). The data sampling rates vary for the different parameters and also depend on aircraft type. It is assumed that proper pre-sampling filters have been applied before recording the data. The basis of all post-processing and calculations is a fixed sampling rate of $1 \mathrm{~Hz}$. For this purpose, data interpolation is required for various parameters.

\section{Wind velocity derivation}

The inertial reference system (IRS) includes two inertial reference units (IRUs), each containing three laser gyros and three accelerometers, and detects angular rates and linear accelerations. The data are resolved to local vertical coordinates and combined with air data inputs to compute the wind speed and direction, among others parameters. Unfortunately this parameter is not available in our database, so we need to derivate it based on parameters at our disposal.

Let us consider an aircraft flying through an airspace region of varying wind speed and direction, both in time and position. The problem we examine here is that of deriving the horizontal wind speed modulus and direction along the flight path starting from flight data measured by on-board instruments or systems.

Depending on the aircraft type and family, available data are ground velocity derived from GPS position measurements, TAS or calibrated air speed 


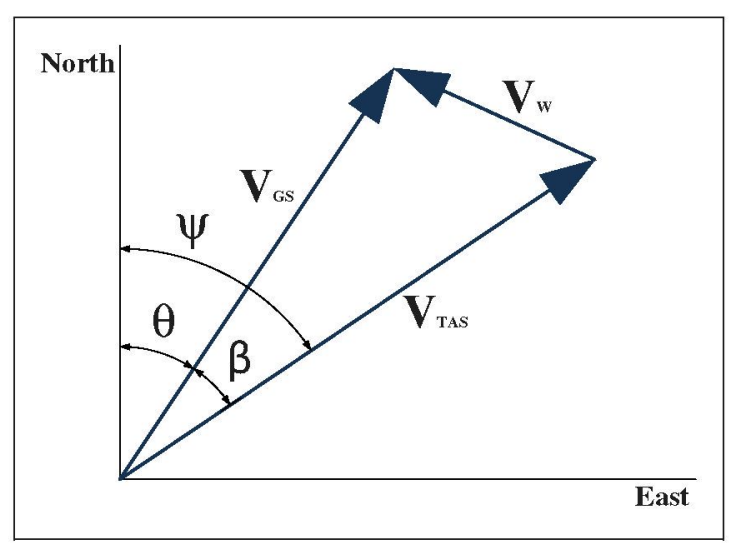

Figure I. The aircraft ground speed $V_{\mathrm{GS}}$ is the vector sum of the aircraft velocity relative to the air $V_{\text {TAS }}$ and the wind velocity $V_{w}$. Heading $\psi$, track angle $\theta$, and crab angle $\beta$ are also shown in the figure.

(CAS), pressure altitude, and temperature. We use the fundamental vector relationship among velocities and their corresponding angles, $\psi$ and $\theta$ (Figure 1)

$$
\overrightarrow{V_{\mathrm{GS}}}=\overrightarrow{V_{\mathrm{TAS}}}+V_{\mathrm{W}}
$$

While the heading angle is available from aircraft on-board instruments $(\psi)$, the track angle $(\theta)$ may be obtained through the $x$ and $y$ coordinates by using the following simple equation

$$
\theta=\tan ^{-1}\left(\frac{\Delta x}{\Delta y}\right)
$$

So that the wind velocity modulus $V_{\mathrm{W}}$ and wind direction $W_{\mathrm{d}}$ with respect to the north are obtained

$$
\begin{aligned}
V_{\mathrm{Wx}} & =\left|\overrightarrow{V_{\mathrm{GS}}}\right| \cdot \operatorname{sen} \theta-\left|\overrightarrow{V_{\mathrm{TAS}}}\right| \cdot \operatorname{sen} \psi \\
V_{\mathrm{Wy}} & =\left|\overrightarrow{V_{\mathrm{GS}}}\right| \cdot \cos \theta-\left|\overrightarrow{V_{\mathrm{TAS}}}\right| \cdot \cos \psi \\
V_{\mathrm{W}} & =\sqrt{V_{\mathrm{Wx}}^{2}+V_{\mathrm{Wy}}^{2}} \\
W_{\mathrm{d}} & =\tan ^{-1}\left(\frac{W x}{W y}\right)+180^{\circ} \quad\left(0^{\circ} \leqslant W_{\mathrm{d}}<360^{\circ}\right)
\end{aligned}
$$

Where subscripts $x$ (east) and $y$ (north) denote the Cartesian or local components.

When the $V_{\text {TAS }}$ is not provided, such is the case of all the aircraft on the sample; it may be obtained from CAS ( $\left.V_{\mathrm{CAS}}\right)$ or by using atmospheric air data. In the first case, we use the following equation

$$
q_{c}=P_{0}\left\{\left[1+\frac{\gamma-1}{2}\left(\frac{V_{\mathrm{CAS}}}{a_{0}}\right)^{2}\right]^{\frac{\gamma}{\gamma-1}}-1\right\}
$$

Where the atmospheric pressure is obtained from the equation of the troposphere in the International Standard Atmosphere (ISA)

$$
p_{z}=p_{0}\left[1+\frac{\lambda}{T_{0}} z\right]^{\frac{-g_{0}}{R \lambda}}
$$

Therefore, the TAS is

$$
V_{\mathrm{TAS}}=a_{0} \sqrt{\frac{T_{z}}{T_{0}}} \sqrt{\frac{2}{\gamma-1}\left[\left(\frac{q_{c}}{p_{z}}+1\right)^{\frac{\gamma-1}{\gamma}}-1\right]}
$$

Based on atmospheric temperature, $T_{z}$, dynamic pressure, $q_{c}$, derived from $V_{\mathrm{CAS}}$, and the atmospheric pressure, $p_{z}$, given by the pressure altitude. While in the second case, the $V_{\text {TAS }}$ is obtained using the following equation

$$
V_{\mathrm{TAS}}=a_{z} M=\sqrt{\gamma R T_{z}} M=a_{0} \sqrt{\frac{T_{z}}{T_{0}}} M
$$

Where the variables needed were atmospheric air temperature, $T_{z}$, and Mach number, $M$.

\section{Filtering and smoothing process of the raw data used in the test}

Before introducing the corresponding variables in the aforementioned formulae, some of them need to be pre-processed. FDR information used in this paper provides raw data that has to be processed before being used in the test presented below.

On the one hand, raw data provided by the airline present outliers in some of the variables (e.g. longitude and latitude, Figure 2), therefore some kind of filtering is required. An ad-hoc filtering has been created for this purpose. First of all, data for the first eight points have been checked to see if there were out of range. Outliers have been removed and replaced by new points obtained by means of a first degree polynomial curve fitting. Second of all, the whole curve has been filtered by using a linear interpolator based on the eight previous measurements.

On the other hand, variables such as longitude, latitude, radio altitude, and pressure altitude have a data sampling rates of 2 or $4 \mathrm{~s}$, depending on the aircraft type. In those cases, variable values are repeated every 2 or $4 \mathrm{~s}$ giving a stepped curve, consequently some kind of interpolation it is also needed to keep a sampling rate of $1 \mathrm{~Hz}$. Moreover, radio altimeter stored data present in many cases shows a stepped aspect that has been also corrected with this filter.

In order to smooth the signal different types of filters were tested. Eventually we chose a 15-point moving average filter with a central moving average because this type of filters are appropriate when the data are collected rapidly compared with their change, are optimal for reducing random noise while retaining a sharp step, and they have a linear-phase response avoiding signal delay. The number of window points is a trade-off between increasing the signal-to-noise and the signal distortion. ${ }^{18}$ 


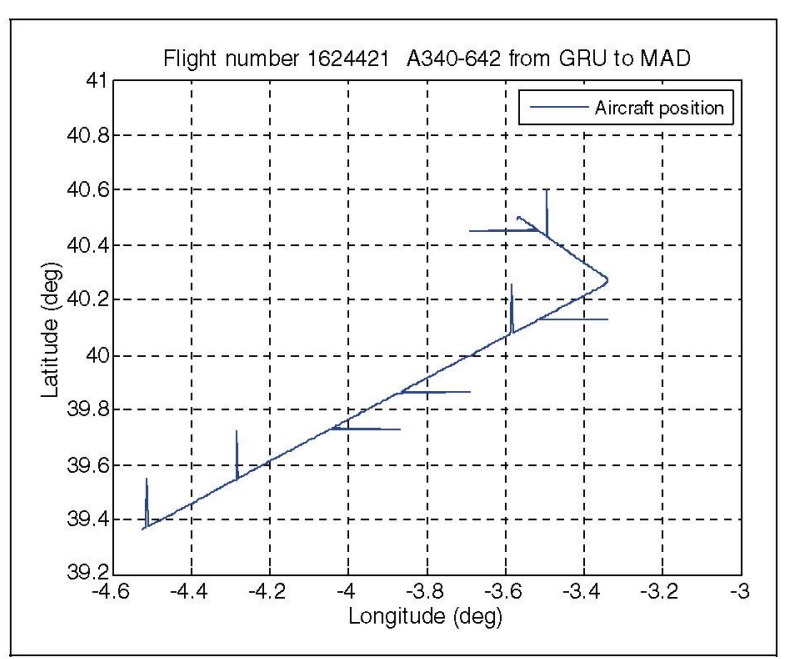

Figure 2. The longitude and latitude in certain aircraft data present some outliers which were removed.

An additional problem arose when deriving the track angle. In this case, despite the fact that $x$ and $y$ data were previously filtered, the track angle needed to be filtered twice before to be usable in wind estimation. By using

$$
\theta_{n}=\operatorname{arctg}\left(\frac{x_{n+1}-x_{n}}{y_{n+1}-y_{n}}\right) \quad\left(0^{\circ} \leqslant \theta<360^{\circ}\right)
$$

The final appearance of the wind velocity was as shown in Figure 3.

The figure shows that both wind speed and wind direction exhibit abnormal changes during turns. This is due to various factors. One of them appears when the track angle and heading are close to $\pm 180^{\circ}$. In this case, small increments in those variables provide a great difference between them making large variations in wind velocity, modulus, and direction. This drawback is clearly depicted in Figure 4, where heading and track angle are drawn for an aircraft after touchdown. Other limitations regarding wind calculations during turns will be accounted for in "Limitations" sub-section.

Regarding Figure 4, we also tried to estimate the wind after the landing, when heading and track angle should match, however we found two obstacles. First, we were unable to obtain accurate $V_{\text {TAS }}$ because minimum Mach Number recorded is 0.1 so that when obtaining $V_{\text {TAS }}$ through the Mach number and the atmospheric temperature, it remains constant from the runway to the aircraft stop.

In addition, when $V_{\mathrm{GS}}$ is low, increments of $x$ and $y$ are very low, and this fact brings great errors in the estimation of the track angle.

\section{Limitations}

The process of deriving wind speed by using the ground speed and the TAS is already performed onboard. Actually, wind speed and direction are already

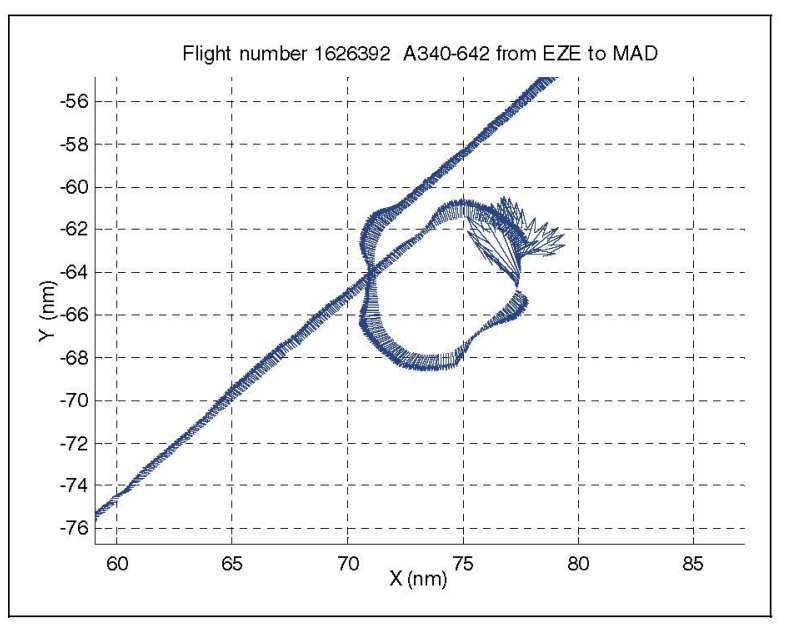

Figure 3. The estimated wind velocity shows a weird modulus and direction during turns.

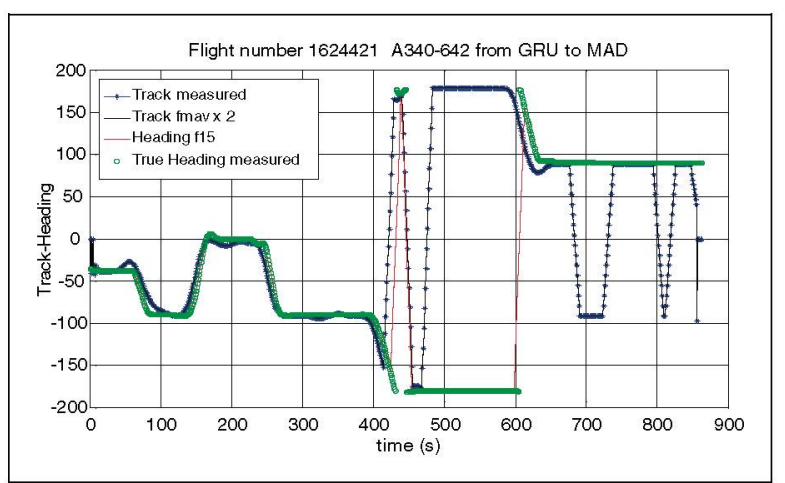

Figure 4. Aircraft flying with heading near $180^{\circ}$ provide great wind modulus due to angle small changes.

presented to the cabin crew members in the navigation display (ND) by the air data inertial reference (ADIR) system. Nevertheless, Airbus documentation on this very system states that there are two aspects that need to be considered with regard to the accuracy of wind information:

- During yaw movements, wind value seen on the NDs could be different than the one displayed by the ADIR unit.

- Wind speed and direction displayed to the crew members have to be used with care regarding GS and TAS accuracy

GS accuracy : \pm 8 knots. TAS accuracy : \pm 4 knots Wind ( $>50$ knots) accuracy : $\pm 12 \mathrm{knots}$ and $\pm 10^{\circ}$

True track : $\pm 2.3^{\circ}$ with $\mathrm{GS}=200$ knots

True heading : $\pm 0.4^{\circ}$

It should be noted that the precision on the value of the wind is not given for wind speeds below 50 knots. $^{19}$ 
According to this report, in the worst-case scenario, when the wind velocity is about 50 knots, the error considered by Airbus represents $25 \%$ of the nominal value, and slightly lower percentage for higher wind speeds. Given the fact that the wind speed accuracy has been not provided by the aircraft manufacturer, we will assume that we will not be able to reach much better values for wind speeds under 50 knots. Furthermore, we can represent the value of the wind velocity modulus for different values of $V_{\text {TAS }}$ and $\beta$, where the crab angle, is defined as

$$
\beta=\psi-\theta
$$

The value of wind modulus as a function of $\beta$

$$
V_{W}=\sqrt{V_{\mathrm{GS}}^{2}+V_{\mathrm{TAS}}^{2}-2 V_{\mathrm{GS}} V_{\mathrm{TAS}} \cos \beta}
$$

The value of wind velocity modulus obtained by using the propagation of uncertainties equation for wind modulus as a function of $V_{\mathrm{GS}}, V_{\mathrm{TAS}}$, and $\beta\left[V_{W}=f\left(V_{\mathrm{GS}}, V_{\mathrm{TAS}}, \beta\right)\right]$

$$
\left|\Delta V_{W}\right|=\left|\frac{\partial f}{\partial V_{\mathrm{GS}}}\right|\left|\Delta V_{\mathrm{GS}}\right|+\left|\frac{\partial f}{\partial V_{\mathrm{TAS}}}\right| \Delta V_{\mathrm{TAS}}|+| \frac{\partial f}{\partial \beta}|| \Delta \beta \mid
$$

Where $\Delta \beta$ has been obtained as a function of $\psi$ and $\theta[\beta=g(\psi, \theta)]$

$$
|\Delta \beta|=\left|\frac{\partial g}{\partial \psi}\right||\Delta \psi|+\left|\frac{\partial g}{\partial \theta}\right||\Delta \theta|
$$

Figure 5 shows wind uncertainties for three crab angles: $0^{\circ}, 10^{\circ}$, and $20^{\circ}$. As we can see, the range of wind modulus values is wider for lower values of $\beta$, reaching the trivial value of zero when $V_{\mathrm{GS}}$ and $V_{\mathrm{TAS}}$ have the same modulus, direction, and sense. For each of the curves represented in Figure 5, we have plotted the uncertainty associated with the wind modulus value.

It may be noticed that the $\beta=0$ curve has no uncertainty bar for wind modulus equal to zero, this is due to the cancelation of all terms included on the propagation of the uncertainty equation.

Minimum and maximum values of wind modulus uncertainty are 8.856 and $9.618 \mathrm{kt}$, respectively. Average values of wind velocity modulus and maximum wind uncertainty with its corresponding $V_{\mathrm{TAS}}$ have been listed in Table 1.

Data show that while the average wind modulus is significantly increasing with $\beta$ (wind speed is over $50 \mathrm{kt}$ for $\beta$ equal to or greater than $15^{\circ}$ ) maximum uncertainty is slightly greater than the initial one (and its value is attained at increasing $V_{\mathrm{TAS}}$ ) representing a lower percentage of the wind modulus.

Assuming the true track uncertainty value is constant and following the same process for different $V_{\mathrm{GS}}$ $(150$ and $250 \mathrm{kt})$ the maximum wind uncertainty

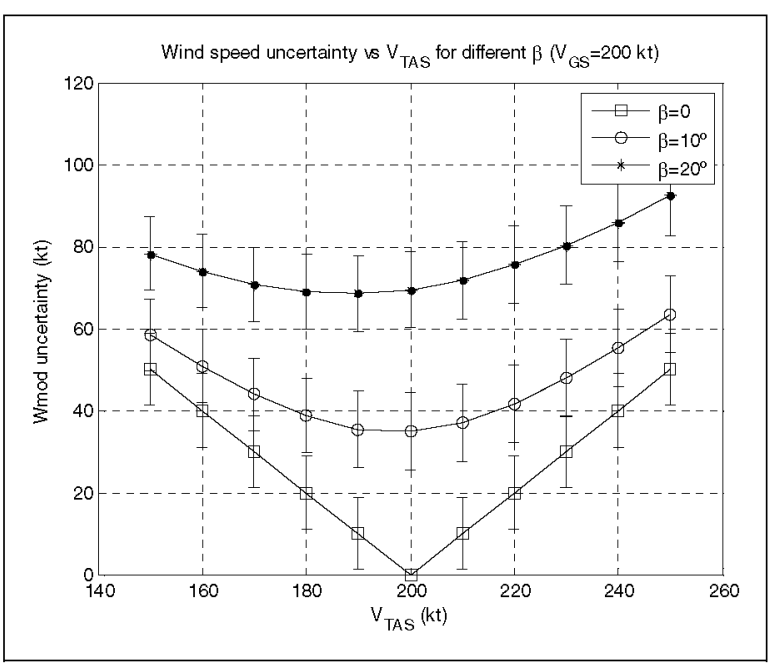

Figure 5. Estimated wind velocity modulus and its uncertainty for $V_{\mathrm{GS}}=200 \mathrm{kt}, \beta=0^{\circ}, 10^{\circ}$, and $20^{\circ}$, and $V_{\mathrm{TAS}}$ from 150 to $250 \mathrm{kt}$.

Table I. Average values of wind modulus and maximum wind modulus uncertainty at its corresponding $V_{\text {TAS }}$ for given $\beta$ values.

\begin{tabular}{rlll}
\hline$\beta()$ & Average $V_{W}(\mathrm{kt})$ & $\begin{array}{l}\text { Maximum } \pm \varepsilon_{V_{W}} \\
(\mathrm{kt})\left(V_{\text {TAS }}\right)\end{array}$ & $\%$ \\
\hline 0 & 27.273 & $8.944($ all $)$ & 32.80 \\
5 & 33.936 & $9.424(200)$ & 27.77 \\
10 & 46.130 & $9.485(210)$ & 20.56 \\
15 & 60.546 & $9.549(230)$ & 15.77 \\
20 & 75.966 & $9.618(240)$ & 12.66 \\
\hline
\end{tabular}

obtained is 8.944 and $11.781 \mathrm{kt}$, respectively, which is in line with Airbus data.

The same process may be followed with wind direction; nevertheless we should keep angles, $\psi$ and $\theta$, through the calculations to obtain wind direction uncertainty.

To sum up, we may infer two important conclusions: first, wind values obtained during yaw movements are not reliable, agreeing with Airbus information and also supported by our calculations; and second, from the above analysis we may infer that the wind velocity modulus uncertainty is in the range of 9 and $12 \mathrm{kt}$ for $V_{\mathrm{GS}}$ and $V_{\mathrm{TAS}}$ between 150 and $250 \mathrm{kt}$, representing a significant percentage of the average wind modulus.

\section{Reference models for atmospheric behavior}

Meteorological services are responsible for studying both at national and international levels the state and behavior of the Earth's atmosphere, its interaction with the oceans and the climate, in general. 
In Spain, the authority responsible for the meteorological service is the so called "Agencia Estatal de Meteorologia" (AEMET), ${ }^{20}$ with the primary intention of developing, implementing, and providing meteorological services and to contribute towards safety regularity an efficiency of international air navigation. Among others, they take and record meteorological observations in Spain, monitor and forecast weather and they also do scientific research in NWP models.

NWP models use the equations of fluid dynamics and thermodynamics to estimate the state of the atmosphere at some time in the future at given locations and altitudes. Observation data are entered into the model to generate initial conditions. These inputs are observations on the one hand from radiosondes located in weather balloons that measure various atmospheric parameters and transmits them to a fixed receiver, and on the other hand from weather satellites. Some of these inputs come from Earth surface observations (synop), another from different altitudes (temp), from meteorological satellites (satob), from commercial aircraft (airep), etc. (Figure 6).

Due to diverse spatio-temporal data acquisition, these observations need to be processed by data assimilation and objective analysis methods, so as to obtain values at locations usable by the meteorological model. This is the way of obtaining initial values for the grid which will be used in the model as the starting point for a forecast; this process is referred to as analysis.

In order to get meteorological data in the future at different locations, we have to analyze the movement of atmospheric air. It responds to general fluid equations, i.e. the equations of fluid dynamics and thermodynamics. The former are the Navier-Stokes equations, an application of Newton's second law, conservation of momentum, which are nonlinear

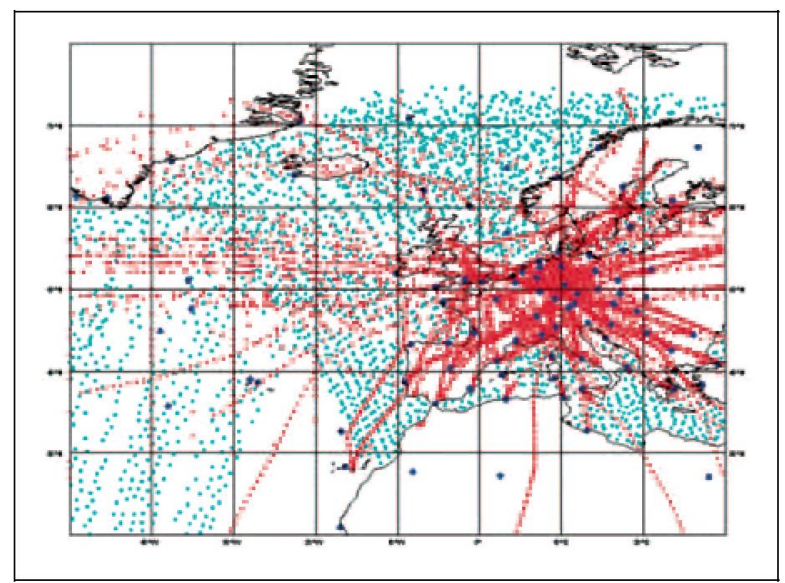

Figure 6. Distribution of assimilated meteorological observations from satellite (light blue), aircraft (red), and radiosondes (dark blue). Information elaborated by the Agencia Estatal de Meteorología, Ministry of Agriculture, Food and Environment. partial differential equations, impossible to solve exactly through analytical methods; the latter are mass and energy conservation, along with the ideal gas law. These equations are known as the primitive equations.

In an inertial frame of reference, and for a spherical coordinate system, the general form of the equations of fluid motion may be written for an arbitrary portion of the fluid as follows

$$
\frac{D \boldsymbol{v}}{D t}=\frac{\partial \boldsymbol{v}}{\partial t}+\boldsymbol{v} \cdot \nabla \boldsymbol{v}=-\frac{1}{\rho} \nabla p-\nabla \phi-2 \boldsymbol{\Omega} \wedge \boldsymbol{v}+\boldsymbol{f}_{\boldsymbol{r}}
$$

The mass conservation equation

$$
\frac{\partial \rho}{\partial t}+\nabla \cdot(\rho \boldsymbol{v})=0
$$

The first law of thermodynamics

$$
\dot{Q}=c_{p} \frac{d T}{d t}-\frac{1}{\rho} \frac{d p}{d t}
$$

The ideal gas law

$$
\frac{p}{\rho T}=R
$$

They are nonlinear partial differential equations that require to be solved using numerical methods. While some models use finite differences, in which the world (or a limited part of it) is represented as discrete points on a regularly spaced grid of latitude and longitude; others use spectral methods that are solved for a range of wavelengths.

Based on the aforementioned meteorological observation and on variables forecasted, airport meteorological stations worldwide provide different reports for air navigation purposes.

The AEMET provides forecasted information through HIRLAM, ${ }^{20,21}$ a grid-point hydrostatic model with a semi-Lagrange dynamic scheme, where radiative processes and those occurring at too smallscale are parameterized (turbulence, clouds and condensation, convection, water and energy exchanges with the surface, etc).

HIRLAM runs four times a day in AEMET in three distinct domains: Euro-Atlantic area with $16 \mathrm{~km}$ horizontal resolution and two focused on the Iberian Peninsula and the Canary Island with $5 \mathrm{~km}$ resolution. The number of vertical levels is 40 . Wind fields, 10-m-height winds, provided for the global model of the European Centre for Medium-Term Forecast (ECMWF) are received four times a day and are used to force the model with five wind analyzed fields: $0,03,06,09$, and $12 \mathrm{~h}$. The observation data, received thanks to the global telecommunications system set up by the World Meteorological 
Organization (WMO), are used to determine the atmospheric initial state regularly.

In Madrid-Barajas airport, half-hourly routine meteorological reports METAR and aerodrome special meteorological reports (SPECI), short Terminal Aerodrome Forecast (TAF) and long TAF, SIGMET, and AIRMET, are supplied as well as aerodrome warnings via data link or in some cases broadcasted. ${ }^{22}$

In Spain, sort TAF reports, for instance, have a validity period of $9 \mathrm{~h} ; 24$ or $30 \mathrm{~h}$ for long TAFs, meteorological aeronautical products (PAM), consisting of information for different flight levels and certain points, given by latitude and longitude, of a series of direct meteorological parameters (geopotential height, wind, temperature, etc.), among others, with analysis and predictions are provided for $6,12,24,36$ and $48 \mathrm{~h}$.

Even when forecasted meteorological conditions are generally not far away from real variables measured, the use of aircraft wind data as input in our algorithm opens the door to the possibility of having available an algorithm providing precise, quantitative and real-time winds information.

Although the original intention was to apply the Navier-Stokes equations, we soon found that there was not enough information within the available data to do it, not even to use the coarse approximation of geostrophic wind model. This model establishes the equilibrium between the pressure gradient force and the Coriolis force, the latter due to the movement of the Earth's frame. This is to say, the geostrophic model neglects the effect of friction between the air and the land, and assumes the system is stationary.

$$
\begin{aligned}
& \frac{D u}{D t}=0=-\frac{1}{\rho} \frac{\partial p}{\partial x}+f \cdot v \\
& \frac{D v}{D t}=0=-\frac{1}{\rho} \frac{\partial p}{\partial x}-f \cdot u \\
& \frac{D w}{D t}=0=-g-\frac{1}{\rho} \frac{\partial p}{\partial z}
\end{aligned}
$$

Even though some of the variables we needed were available on-board (i.e. geometric altitude, vertical geometric acceleration), they were not provided in FDR data furnished to us, and therefore we were not be able to apply the above approximation. This led us to the application of a particular interpolation method.

\section{Chosen interpolation method}

As wind data are recorded at different point locations, a spatial interpolation method is required to estimate data values at other locations. In general, the higher the point density is, the better the results are. But some variables, such as wind, present a wider variation over shorter distances than temperature or relative humidity. ${ }^{23}$ Likewise the spatial arrangement of these points can take different forms and, as a result, the solution surface will be highly form-dependent, as the error. This means that we would require a denser grid of points but also the spread of points will be crucial to achieve an accurate and precise solution surface.

Interpolation methods are generally classified into deterministic and geostatistical methods. ${ }^{24}$ The first ones assign values to locations based on the surrounding measurements and use specified mathematical formulae that will determine the smoothness of the resulting surface. Some examples of this method are inverse distance weighting (IDW), natural neighbor, trend, and spline.

The second one, geostatistical methods are based on statistical models that take into account the autocorrelation, it is to say, the statistical relationship among the measured points. Because of this, geostatistical techniques not only have the capability of producing a solution surface but also provide some measure of the certainty or accuracy of the surface. May be the most representative example of geostatistical method is the Kriging method.

Interpolation methods may also be classified into exact and inexact methods. The former are used when the known values exactly match the interpolation surface, while the latter, inexact methods, do not establish this constraint, and therefore provide a smoother solution.

Those methods can also be divided into other two types: global and local. Global interpolators use all the available data to provide a solution surface, but local methods use only the information surrounding the point being estimated. The Kriging methods are local (exact and stochastic) interpolators that use the more advanced geoestatistical techniques.

Some comparisons among several interpolation techniques have been performed in different studies, ${ }^{23,25}$ with diverse results depending on the spatial distribution of the data.

In this study, we were looking for a deterministic method, and we are neither interested in the probability of having a certain value exceeded nor in the probability of having the interpolated value within a range, therefore we put aside geostatistical methods.

Moreover, we did not need an exact method, meaning the surface solution verifying the data set, because each known wind point has been obtained as a weighted mean value in a particular cell; and on the other hand, we avoided exact methods for the sake of having a smoother surface. Finally, in order to be less time-consuming (pre-processing sometimes takes a while, depending on the number of aircraft involved) we decided to choose a mesh-less method. Furthermore, taking into consideration the variability and size of the data set and the extent of the studied area, we resort to deterministic, inexact, global method: a trend surface analysis, in spite of the fact 
that deterministic methods provide no indication of the extent of possible errors.

\section{One-dimensional formulation}

The goal of this technique is to find a trial function $\hat{u}(x, y)$, within a class of functions, polynomials in this case, that best fits the data set $\left(x_{i}, y_{i}, V_{w i}\right)$. Given the trial function defined as

$$
\hat{u}(x, y)=\sum_{i=1}^{m} p_{i}(x, y) a_{i}(x, y)=p^{T}(x, y) a(x, y)
$$

Where

$$
\hat{u}(x, y) \text { is the approximation to function } u(x, y) \text {, }
$$$$
p^{T}(x, y)=\left(p_{1}(x, y), p_{2}(x, y), \ldots, p_{m}(x, y)\right)
$$

is the basis function vector

$$
a^{T}(x, y)=\left(a_{1}(x, y), a_{2}(x, y), \ldots, a_{m}(x, y)\right)
$$

is the corresponding coefficient vector

$m$ is the number of terms in the basis function vector.

The linear and quadratic function vectors are given, respectively, by

$$
\begin{aligned}
& p^{T}(x, y)=(1, x, y) \\
& p^{T}(x, y)=\left(1, x, y, x^{2}, x y, y^{2}\right)
\end{aligned}
$$

With a number of terms, $m$, of 3 and 6 , respectively.

The measurement residual for a known point is given by the difference between the variable value at any given point and the approximation function at that point

$$
\begin{aligned}
\varepsilon_{u_{I}} & =u\left(x_{I}, y_{I}\right)-\hat{u}\left(x_{I}, y_{I}\right) \\
& =u\left(x_{I}, y_{I}\right)-p^{T}\left(x_{I}, y_{I}\right) a(x, y)
\end{aligned}
$$

The unknown parameters $a_{i}(i=1,2, \ldots, m)$ are determined minimizing the following cost function

$$
\begin{aligned}
J(u) & =\frac{1}{2} \varepsilon_{u}^{T} \varepsilon_{u}=\frac{1}{2}(u-\hat{u})^{T}(u-\hat{u}) \\
& =\frac{1}{2}(u-P a)^{T}(u-P a)
\end{aligned}
$$

Where $P$ is the observation matrix, for a linear function with $n$ data points

$$
P=\left[\begin{array}{ccc}
1 & x_{1} & y_{1} \\
1 & x_{2} & y_{2} \\
\cdots & \cdots & \cdots \\
1 & x_{n} & y_{n}
\end{array}\right]
$$

Expanding $J(u)$ :

$$
J(u)=\frac{1}{2}\left(u^{T} u-u^{T} P a-a^{T} P^{T} u+a^{T} P^{T} P a\right)
$$

Its minimum with respect to $a$ is found by setting the gradient to zero

$$
\frac{\partial J(u)}{\partial a}=\left(P^{T} P a-P^{T} u\right)^{T}=0
$$

Solving for $a$, the least-square estimator is found to be

$$
a=\left(P^{T} P\right)^{-1} P^{T} u=P^{L} u
$$

Where $P^{L}$ is the left pseudoinverse of $P$.

\section{Two-dimensional formulation}

The process described in previous section was independently applied for both wind components: north and east, so that we had two trial functions

$$
\begin{aligned}
& \hat{u}(x, y)=p_{u}^{T}(x, y) a_{u}(x, y) \\
& \hat{v}(x, y)=p_{v}^{T}(x, y) a_{v}(x, y)
\end{aligned}
$$

Using this approximation procedure, it gave us, for the linear function, six unknown polynomial coefficients; 12 , if the function is a quadratic one, and so forth.

\section{Complex variable formulation}

Nevertheless, our intention was to retain the liaison between both components so we also essay a 1-D complex trial function

$$
\begin{aligned}
\hat{w}(z) & =\hat{u}(z)+i \hat{v}(z)=p^{T}(z) a(z) \\
z & =x+i y
\end{aligned}
$$

The interpolation method leads us to

$$
\begin{aligned}
& \hat{u}(z)=\operatorname{Re}[\hat{w}(z)] \\
& \hat{v}(z)=\operatorname{Im}[\hat{w}(z)]
\end{aligned}
$$

In this case, the number of coefficients to be derived was two for the linear function and three for a quadratic one.

In this manner, reducing the number of unknowns for a specific area with a fixed number of data, we increase the number degrees of freedom which may be obtained by subtracting the number of estimated parameters from the number of observations (data). The higher the number of degrees of freedom is, the higher the precision of estimators is. 


\section{FDR data results}

FDR samples provided by the airline consist of several files containing FDR data for a number of days. We were interested in having a high number of aircraft in the area of Madrid airport so as to increase the interpolation accuracy.

The rate of aircraft landing or taking off at Madrid airport is about 42 per hour (average hourly value for 2012 , with 373,185 operations). ${ }^{26}$ Regrettably, we do not have all the aircraft landing and taking off data available, only those contained in the aforementioned file, thus after analyzing these files we choose the one with a higher number of aircraft at the same time.

Unfortunately, as Figure 7 shows, we got at best only four aircraft flying in Madrid airport area in our samples. It means 4 points per second in a box with horizontal grid of about $100 \times 100 \mathrm{~nm}$ and $8000 \mathrm{~m}$ of altitude.

Taking into account the spatio-temporal correlation of wind, ${ }^{27}$ we have set up a grid of $10 \times 10 \mathrm{~nm}$ in the horizontal plane, $1000 \mathrm{~m}$ of altitude and a 15minute window. Obtaining for each cell the average value of the wind, this simulation resulted in Figure 8 , where there are only eight points known in the map.

Due to the low number of points available, even in the case of grouping wind values for each grid and for a 15 -minute window, we decided to check the validity of this interpolation method using several wind scenarios obtained from a global weather model.

\section{Different wind scenarios}

Three wind conditions are going to be tested for this two interpolation techniques. They are real weather forecasts performed using the US GFS global weather model. Through free software called Ugrib, we downloaded the corresponding grib files containing meteorological data such as longitude, latitude, with a $0.5^{\circ}$ resolution, surface winds, and barometric pressure. This global model provides a 7-day forecast with a 3-h time-step.

\section{Light wind}

This wind map contains a grid made up of $11 \times 9$ points, longitude from 41.8 to $46.7 \mathrm{~W}$; latitude from 24.5 to $28.4 \mathrm{~N}$. Their average wind components values are

$$
\begin{aligned}
u & =2.793 \mathrm{kt} \\
v & =1.842 \mathrm{kt}
\end{aligned}
$$

Its average modulus is $3.553 \mathrm{kt}(6.580 \mathrm{~km} / \mathrm{h})$, a light breeze in the Beaufort scale.

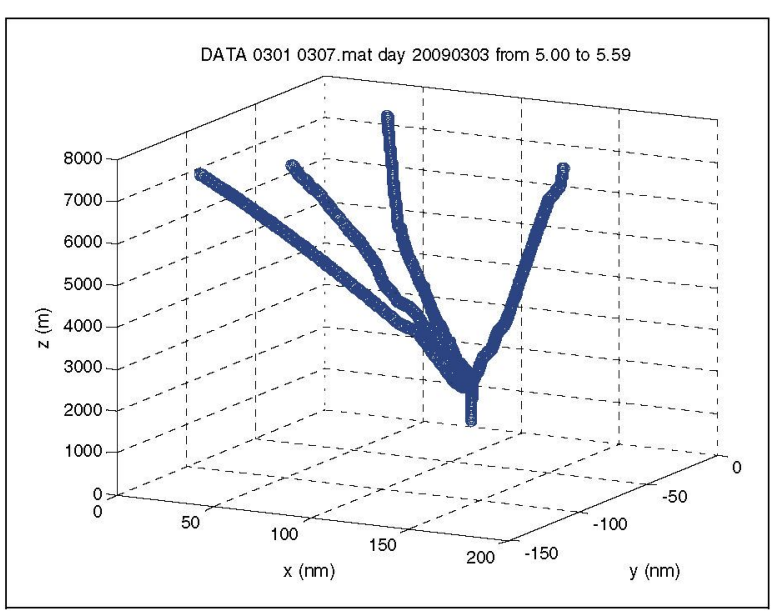

Figure 7. Aircraft landing at Madrid airport on 3 March 2009, from 5.00 to $5.59 \mathrm{~h}$, according to airline data.

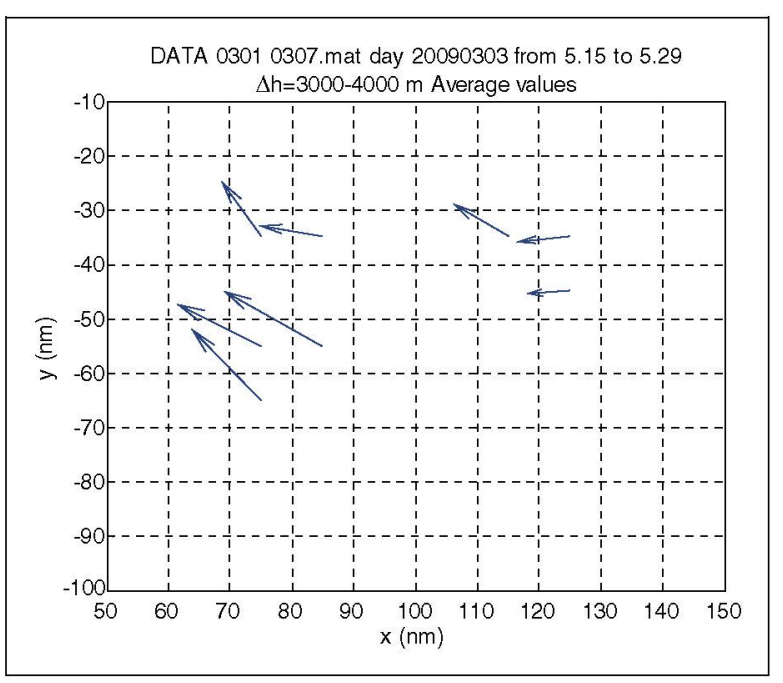

Figure 8. Wind vectors obtained on 3 March 2009, from 5.15 to $5.29 \mathrm{~h}$, within 3000 and $4000 \mathrm{~m}$ of altitude.

\section{Strong wind}

This wind map (Figure 9) contains a $11 \times 9$ points grid, longitude from 11.7 to $17.5 \mathrm{~W}$; latitude from 24.5 to $28.4 \mathrm{~N}$. Average wind components value

$$
\begin{aligned}
u & =28.909 \mathrm{kt} \\
v & =-11.349 \mathrm{kt}
\end{aligned}
$$

Its average modulus is $31.635 \mathrm{kt}(58.589 \mathrm{~km} / \mathrm{h})$, a high wind in the Beaufort scale.

\section{Wind with vorticity}

This wind map contains a $7 \times 7$ points grid, longitude from 11.7 to $17.5 \mathrm{~W}$; latitude from 53.2 to $58.9 \mathrm{~N}$. Average wind components value

$$
\begin{aligned}
u & =3.685 \mathrm{kt} \\
v & =12.770 \mathrm{kt}
\end{aligned}
$$




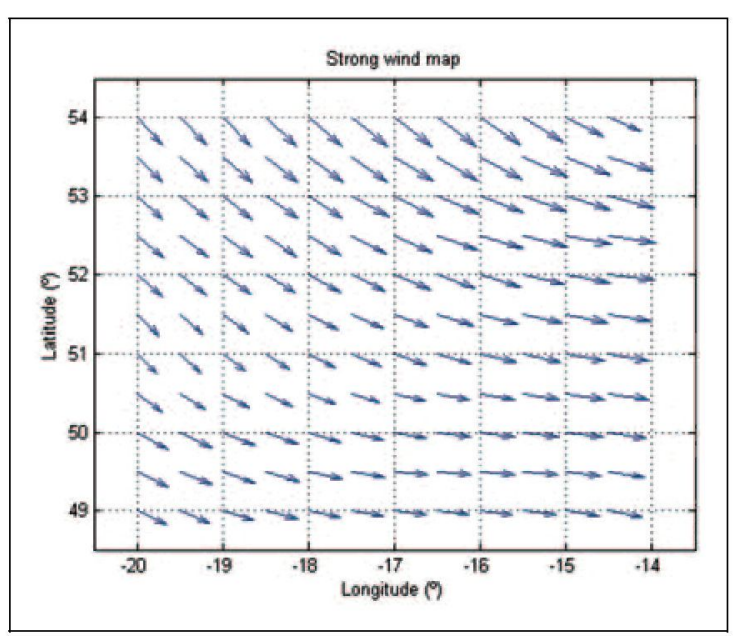

Figure 9. Strong wind map with an average modulus of $29.69 \mathrm{kt}$, where a $1 \mathrm{I} \times 9$ points grid has been selected.

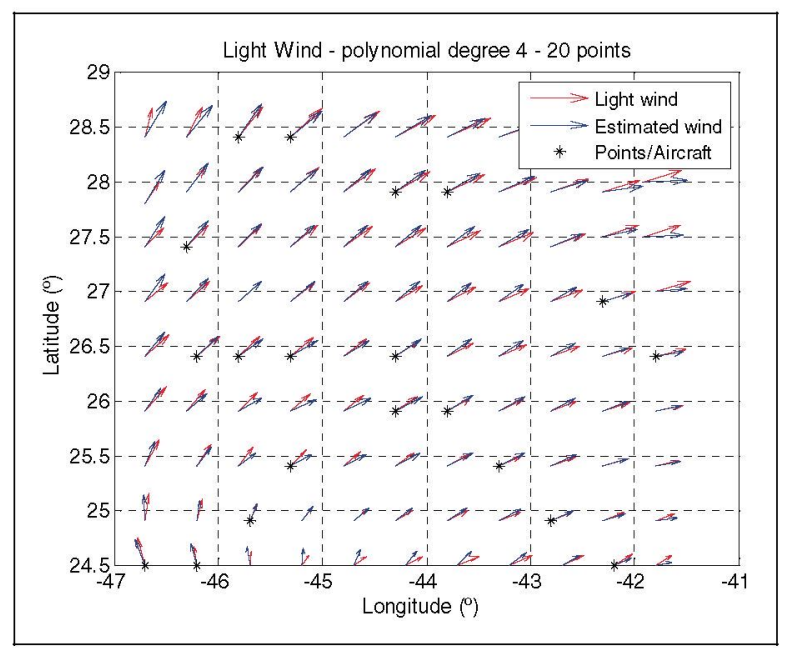

Figure 10. Light wind and estimated wind based on 20 known points.

Its average modulus is $23.410 \mathrm{kt}(43.356 \mathrm{~km} / \mathrm{h})$, a moderate breeze in the Beaufort scale.

\section{Wind scenarios results}

As stated above, not only the number of known points is essential for obtaining a more accurate interpolation, but also their position in the grid. Accordingly we have performed different tests for each scenario. Regarding the number of data points, we carried out 10 - and 20 -point tests and the position of those points have been randomly chosen. The interpolation accuracy has been measured as the distance root mean square (DRMS) according to the following equation

$$
\mathrm{DRMS}=\sqrt{\sigma_{u}^{2}+\sigma_{v}^{2}}
$$

DRMS values have been displayed in Table 2 .

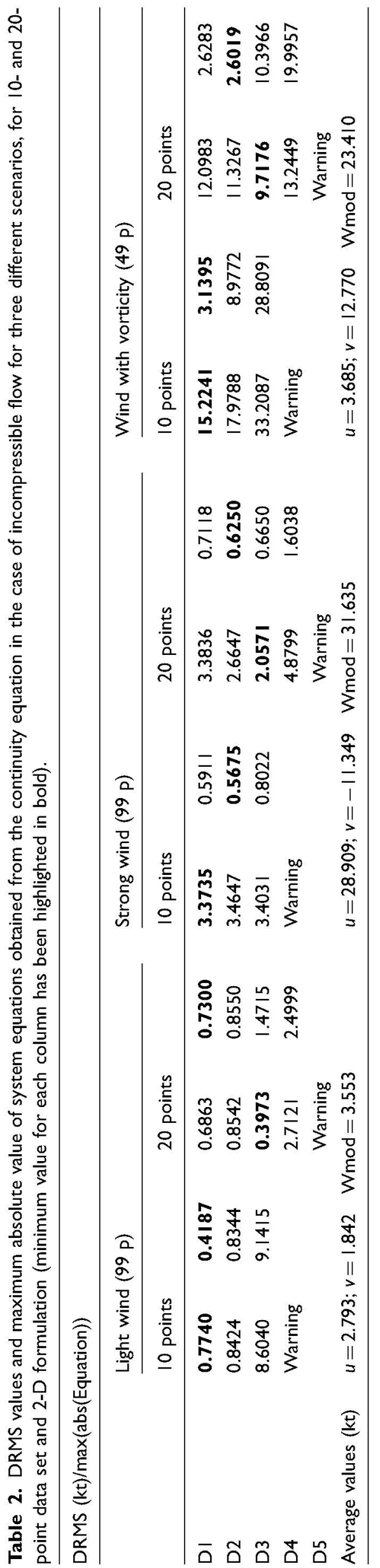


Table 3. DRMS values for three different scenarios, for 10- and 20-point data set and complex variable formulation (minimum value for each column has been highlighted in bold).

\begin{tabular}{|c|c|c|c|c|c|c|}
\hline \multicolumn{7}{|l|}{ DRMS (kt) } \\
\hline & \multicolumn{2}{|c|}{ Light wind $(99 \mathrm{p})$} & \multicolumn{2}{|c|}{ Strong wind $(99 \mathrm{p})$} & \multicolumn{2}{|c|}{ Wind with vorticity $(49 \mathrm{p})$} \\
\hline & 10 points & 20 points & 10 points & 20 points & 10 points & 20 points \\
\hline DI & 0.8325 & 0.6922 & 9.1630 & 10.2883 & $29.8 I 74$ & 26.0181 \\
\hline $\mathrm{D} 2$ & 1.0896 & 0.6998 & 10.2408 & $10.448 \mid$ & 37.4100 & 27.6670 \\
\hline D3 & 0.9024 & 0.7210 & 10.5928 & 10.9368 & 31.3217 & 26.2340 \\
\hline D4 & 1.0790 & 0.6504 & I I.| 479 & $\mid 2.7644$ & & \\
\hline D5 & 1.1959 & 0.7402 & |4.8787 & 12.1876 & & \\
\hline D6 & 2.3135 & 0.9045 & 18.9009 & 22.0173 & & \\
\hline D7 & 2.4552 & 0.9503 & 40.8326 & $|6.53| \mid$ & & \\
\hline $\begin{array}{l}\text { Average } \\
\text { values }(\mathrm{kt})\end{array}$ & \multicolumn{2}{|c|}{$\begin{array}{l}u=2.79931 \\
v=1.8423 \quad W \bmod =3.5532\end{array}$} & \multicolumn{2}{|c|}{$\begin{array}{l}u=28.9091 ; \\
v=11.3486 \quad W \bmod =31.6354\end{array}$} & \multicolumn{2}{|c|}{$\begin{array}{l}u=3.6853 \\
v=12.7697 \quad W \bmod =23.4102\end{array}$} \\
\hline
\end{tabular}

Another measure of atmospheric air behavior, given that none of the fluid mechanics equations was applied due to the lack of the required parameters, was the value the continuity equation in the case of incompressible flow

$$
\nabla \cdot v=0=\frac{\partial u}{\partial x}+\frac{\partial v}{\partial y}
$$

The application of this formula assumes that the divergence of the velocity field is zero in the studied area. This can be an initial hypothesis on grounds of lack of other variables.

For the linear function approximation the equation that should be verified are

$$
\begin{gathered}
\hat{u}(x, y)=a_{0 u}+a_{1 u} x+a_{2 u} y \\
\hat{v}(x, y)=a_{0 v}+a_{1 v} x+a_{2 v} y
\end{gathered}
$$

Thus the resulting equation is

$$
a_{1 u}+a_{2 v}=0
$$

For the quadratic function approximation the equations are

$$
\begin{aligned}
& \hat{u}(x, y)=a_{0 u}+a_{1 u} x+a_{2 u} y+a_{2 u} x^{2}+a_{4 u} x y+a_{5 u} y^{2} \\
& \hat{v}(x, y)=a_{0 v}+a_{1 v} x+a_{2 v} y+a_{2 v} x^{2}+a_{4 v} x y+a_{5 v} y^{2}
\end{aligned}
$$

Equating the corresponding coefficients we obtain this system of equations

$$
\begin{aligned}
a_{1 u}+a_{2 v} & =0 \\
2 a_{3 u}+a_{4 v} & =0 \\
a_{4 u}+2 a_{5 v} & =0
\end{aligned}
$$

Table 2 shows the maximum absolute value of this system of equations for each polynomial approximation degree in the case of independentlyinterpolation components.

As it can be noticed in Table 2, some cells do not provide numerical values but a warning message. It happens because the observation matrix is a 2-D Vandermonde matrix, a matrix whose terms make up a geometric progression in each row. When the polynomial degree increases the matrix becomes ill-conditioned.

The results shown in Table 2 indicate that in the case of a 10 -point set data, the polynomial which provides the minimum DRMS is the linear one for all three of these scenarios. Whereas in the light wind map the DRMS value represents almost $22 \%$ of wind modulus, in the strong wind situation is only $11 \%$ of its corresponding wind modulus. The case of wind with vorticity map has by far the lower accuracy, representing more than $65 \%$ of its mean wind modulus.

Regarding their corresponding system of equations, we can only perform a relative comparison among polynomial of first, second, and third degree; or even among 10- and 20-point data. One issue to take into account is that depending on the equation, the coefficient units are different therefore the comparison is going to be a qualitative one. Looking at the table, the results give a lower value for first degree polynomial in light wind, and a larger one for the wind with vorticity, also in the first degree polynomial.

If we focus on 20-point data, the outcome varies in a significantly manner. First, because the higher accuracy appears in the polynomial of third degree in all three cases, representing more than $11 \%$, $6,5 \%$, and $41 \%$, respectively, of their wind modulus.

With respect to the system of equations, it can be noticed that the greater accuracy is verified for polynomial of first and second degrees, and in no case there is an exact match of the two measurements considered: accuracy and system of equations. 
In addition, when we analyze the outcome of the complex interpolation method, we have assessed the accuracy only through the DRMS because in this case, the coefficients are indeed complex, and the system equation analysis performed in the previous interpolation is not possible. The results are detailed in Table 3.

Some of the cells in Table 3 are empty because in those cases the result is about $100 \%$ of the wind modulus.

Tackling the two wind components as a complex variable, most interesting result is that almost in all the cases, the higher accuracy is verified in linear polynomials. Only for a set data of 20 points in light wind the lower DRMS occurs in a polynomial of fourth degree (Figure 10). Nevertheless, there is no significantly difference between the values obtained in the polynomial of first and fourth degree $(0.6922 \mathrm{kt}$ vs. $0.6504 \mathrm{kt}$ ), each of them representing $18.30 \%$ and $19.48 \%$ of the mean wind modulus, the lowest in the whole table. DRMS in light wind 10-point data is $23.43 \%$ of its wind modulus while the strong modulus shows $28.96 \%$ and $32.52 \%$ of their corresponding 10 - and 20 -point wind modulus. In the particular case of wind with vorticity the results present more than $110 \%$ of their wind modulus.

\section{Discussion}

On the one hand, the analysis of FDR data revealed that many considerations need to be taken when estimating wind speed based on on-board instruments and systems:

- Wind values obtained during yaw movements are not reliable; therefore their corresponding data should be deleted in order to have more accurate wind estimation.

- Wind modulus uncertainty is in the range of 9-12 kt for $V_{\mathrm{GS}}$ and $V_{\mathrm{TAS}}$ between 150 and $250 \mathrm{kt}$, representing a variable percentage of the average wind modulus. For a wider speed range, more analysis would be required.

- Wind speed cannot be obtained using FDR data after touchdown due to low accuracy of Mach number indicator at low speeds.

- Considering a maximum uncertainty allowed of $10 \%$ for the determination of wind speed, we will apply this procedure for $V_{\mathrm{GS}}$ and $V_{\mathrm{TAS}}$ over $120 \mathrm{kt}$, to be on the safe side.

On the other hand, regarding the results of the interpolation methods as a whole, they revealed that the DRMS calculations in the independently-interpolation method indicate that there is a pattern: all the scenarios present a higher accuracy for a first order polynomial in the case of having 10-point data set, while for 20 points, the best accuracy is attaint by using a third degree polynomial. This means that if we are willing to admit errors of $10 \%$ of the average wind modulus we need to have 20 or more aircraft data available for winds without vorticity. The last case, wind with vorticity, needs a deeper study in order to characterize it as it exhibit unacceptable high errors.

Moreover, raw data obtained from the system of equations do not provide useful information about the most accurate polynomial, among other things, because there is no relation among equations results and DRMS.

By the same token, contrary to our expectations, the complex variable not only has not improved the independently-interpolation method as it happens in, ${ }^{28}$ but the results show higher DRMS in all studied cases.

\section{Conclusions}

Firstly, results from the analysis performed on the wind modulus uncertainty based on the manufacturer information shows a value of 9-12 kt when deriving wind velocity modulus from ground speed and TAS, which is in line with the one obtained by Airbus.

Secondly, the data provided by the air carrier are insufficient, making difficult to carry out an extensive study of the wind field. This is due to several reasons: the fact that data are provided only for landing aircraft, the lack of a high number of aircraft information, the separation needed to avoid potential conflicts, etc. This leaded us to test the chose interpolation method using different wind scenarios.

Despite the fact that the complex variable leastsquare approximation method has an explicit physical meaning and greater time-consuming it appears to be less efficient and less accurate than 2-D formulation.

By contrast, the independently-interpolation method has been proven to have a higher accuracy and a lower time consuming than the complex variable interpolation method, for all three scenarios tested and also both 10 - and 20-point set data. However, there is no single polynomial degree which best matches all the scenarios and data sets, reflecting a noticeable variation as a function of the number of points in the sample.

Comparing the results from the three scenarios, we can infer that the higher the wind modulus, the better the precision. Besides, the complexity of the wind map, including air flow of great magnitude, moving in circular paths around a low pressure center (wind with vorticity) provide the worst results for both 10 and 20-point set, which evidence the difficulty of having a good performance by using polynomial approximation.

More research needs to be undertaken to make these preliminary findings into more solid results, to increase the number of FDR data available, and to assay specific functions for the interpolating methods so as to accurately fit air vorticity conditions. 


\section{Funding}

This research received no specific grant from any funding agency in the public, commercial, or not-for-profit sectors.

\section{Conflict of interest}

None declared.

\section{References}

1. SESAR. SESAR and research. http://www.eurocontrol. int/content/sesar-and-research (2009, accessed July 2013).

2. FAA. Next generation air transportation system (NextGen). http://www.faa.gov/nextgen/ (2013, accessed July 2013).

3. Enea $\mathrm{G}$ and Porreta M. A comparison of 4D-trajectory operations envisioned for Nextgen and SESAR, some preliminary findings. In: 28 th Congress of the International Council of the Aeronautical Sciences, 23-28 September 2012, Brisbane, Australia. Paper ICAS 2012-10.3.1.

4. Weiss SJ, Pyle ME, Janjic Z, et al. The operational high resolution window WRF model runs at NCEP: advantages of multiple model runs for severe convective weather forecasting. In: 24 th conference on severe local storms, 27-31 October 2008, Savannah, GA. http://www.spc.noaa.gov/publications/weiss/wrf-hrw. pdf.

5. Oslo CTM3 model from ECMWF, http://folk.uio.no/ asovde/osloctm3/metdata.html (2012, accessed July 2013).

6. Zhang N, Millane RP and Hunter AJ. Estimating wind velocity from limited aircraft flight data. In: Industrial Mechatronics and Automation (ICIMA), 2010 2nd International Conference on. 30-31 May 2010; 2: 626-629.

7. Weng CT, Ho CS, Lan CE, et al. Aerodynamic analysis of a jet transport in windshear encounter during landing. $J$ Aircraft 2006; 43(2): 419-427.

8. Ho CS, Yang MH, Lin PH, et al. Aerodynamic derivatives and wind field estimation in a flight accident involving cross wind. In: Mechanical and Electronics Engineering (ICMEE), 2010 2nd International Conference on. 1-3 August 2010; 1: 271-275.

9. Haverdings $\mathrm{H}$ and Chan PW. Quick access recorder data analysis software for windshear and turbulence studies. $J$ Aircraft 2010; 47(4): 1443-1447.

10. Lan $\mathrm{CE}, \mathrm{Wu} \mathrm{K}$ and $\mathrm{Yu}$ J. Flight characteristics analysis based on QAR data of a jet transport during landing at a high-altitude airport. Chin $J$ Aeronaut 2012; 25(1): 13-24.

11. Delahaye D and Puechmorel S. Aircraft local wind estimation from radar tracker data. In: Control, automation, robotics and vision, 2008. ICARCV 2008. 10th International Conference on 17-20 December 2008: 1033-1038.

12. Hollister WM, Bradford ER and Welch JD. Using aircraft radar tracks to estimate winds aloft. Lincoln Lab $J$ 1989; 2(3): 555-565.

13. Schwartz BE, Benjamin SG, Green SM, et al. Accuracy of RUC-1 and RUC-2 wind and aircraft trajectory forecasts by comparison with ACARS observations. Weather Forecast 2000; 15: 313-326.
14. Benjamin SG, Barry ES and Rodney EC. Accuracy of ACARS wind and temperature observations determined by collocation. Weather Forecast 1999; 14: 1032-1038.

15. Cole RE, Green S, Jardin M, et al. Wind prediction accuracy for air traffic management decision support tools. In: Proceedings of the 3rd USA/Europe Air Traffic Management $R \& D$ Seminar. Napoli, 13-16 June 2000.

16. Alonso Alarcón JF, Sáez Nieto FJ and GarcíaHeras Carretero J. Aircraft used as a sensor for atmospheric behaviour determination. Practical case: pressure estimation using automatic dependent surveillance-broadcast. Proceedings of the Institution of Mechanical Engineers, Part G: Journal of Aerospace Engineering. Epub ahead of print 3 April 2012. DOI: $10.1177 / 0954410012442044$.

17. ARINC Mark 33 Digital Information Transfer System (DITS) part 1. Functional description, electrical interface, label assignments and word formats. ARINC specification 429 part 1-17. Published 17 May 2004.

18. Smith SW. The scientist and engineer's guide to digital signal processing. San Diego, CA: California Technical Publishing, 1997.

19. Aviation investigation report A05H0002. "Runway overrun and fire Air France Airbus A340-313 FGLZQ Toronto/Lester B. Pearson International Airport, Ontario 02 August 2005". Minister of Public Works and Government Services Canada 2007. http://www.tsb.gc.ca/eng/rapports-reports/ aviation/2005/a05h0002/a05h0002.pdf

20. Aviation investigation report A05H0002. "Runway overrun and fire Air France Airbus A340-313 FGLZQ Toronto/Lester B. Pearson International Airport, Ontario 02 August 2005". Minister of Public Works and Government Services Canada 2007. http://www.tsb.gc.ca/eng/rapports-reports/aviation/ 2005/a05h0002/a05h0002.pdf

21. HIRLAM. http://www.hirlam.org/ (accessed July 2013).

22. Guía de servicios MET para la navegación aérea. 6th ed. Madrid: Agencia Estatal de Meteorología, Ministerio de Medio Ambiente, y Medio Rural y Marino, November 2012. http://www.aemet.es/es/conocermas/aeronautica/detalles/Guia_de_servicios_MET

23. Luo W, Taylor MC and Parker SR. A comparison of spatial interpolation methods to estimate continuous wind speed surfaces using irregularly distributed data from England and Wales. Int J Climatol 2007; 28(7): 947-959.

24. Burrough PA and McDonnell RA. Principles of geographical information systems. Oxford: Oxford University Press, 1998.

25. Chang WY, Dai XG and Chen HW. A case study of geostatistical interpolation in meteorological fields. Chin J Geophys 2004; 47(6): 1104-1112.

26. AENA aeropuertos. http:/estadisticas.aena.es/ (accessed July 2013)

27. Chaloulos $\mathrm{G}$ and Lygeros J. Effect of wind correlation on aircraft conflict probability. $J$ Guidance Control Dyn 2007; 30(6): 1742-1752.

28. Cheng YM, Wang JF and Bai FN. A new complex variable element-free galerking method for twodimensional potential problems. Chin Phys B 2012; 21(9): 090203. 


\section{Appendix}

\section{Notation}

$\begin{array}{ll}a_{0} & \text { speed of sound at msl } \\ a_{z} & \text { speed of sound at altitude } z \\ c_{p} & \text { specific heat at constant pressure } \\ f & \text { Coriolis parameter } \\ f_{r} & \text { viscosity force } \\ g_{0} & \text { acceleration of gravity at msl } \\ M & \text { Mach number } \\ p & \text { fluid pressure } \\ p_{0} & \text { pressure at } \mathrm{msl} \\ p_{z} & \text { pressure at altitude } z \\ Q & \text { thermal energy } \\ q_{c} & \text { dynamic pressure } \\ R & \text { gas constant for air } \\ T & \text { fluid temperature } \\ T_{0} & \text { temperature at msl } \\ T_{z} & \text { temperature at altitude } z \\ u & \text { Northern component of wind speed }\end{array}$

Eastern component of wind speed

fluid velocity vector

calibrated air speed

ground speed

true airspeed

wind speed modulus

wind speed direction

position coordinates

crab angle

adiabatic index

increment of $x\left(\Delta x_{i}=x_{i+1}-x_{i}\right)$

increment of $y\left(\Delta y_{i}=y_{i+1}-y_{i}\right)$

track angle

tropospheric lapse rate (ISA)

fluid density

effective potential function

true heading angle

Earth angular velocity 